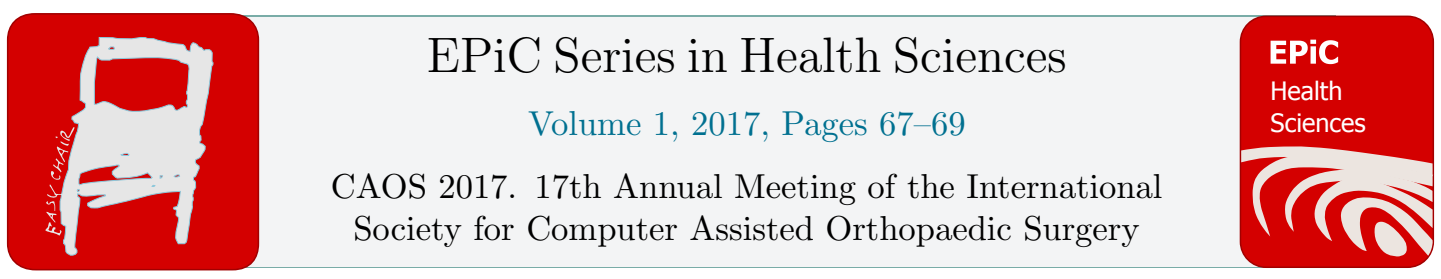

\title{
Fluoroscopy-based Laser Projection System for Surgical Guidance and Its Calibration Methods
}

\author{
Sanghyun Joung ${ }^{1}$, Hyunwoo Lee ${ }^{1}$, Chul-woo Park ${ }^{1}$, \\ Chang-Wug $\mathrm{Oh}^{1,2}$ and Il-Hyung Park ${ }^{1,2}$ \\ ${ }^{1}$ Medical Device and Robot Institute of Park, Kyungpook National University, Daegu, 41566, \\ South Korea \\ ${ }^{2}$ Department of Orthopedic Surgery, Kyungpook National University Hospital, Daegu, 41566, \\ South Korea \\ shjoung@mdrip.knu.c.kr
}

\begin{abstract}
We have developed a laser projection system, which can project laser on corresponding position to surgical planning drawn at a fluoroscopic image without an optical tracking system. In this paper, we introduce a spatial calibration method between a laser module and a fluoroscope for the laser projection and evaluate its accuracy with a mimic experimental system. The experimental system consists of a laser module, a distance measurement unit and a CCD camera. The laser modules can project arbitrary line on surface by reflecting a point source laser with two galvanometers. We designed a calibration phantom by combining a collimator for accurate laser pattern positioning and stainless steel ball arrays for calculation of an extrinsic parameter of a C-arm fluoroscopy. We set a projection plane having ruler in $400 \mathrm{~mm}$ distance from the CCD camera, and set 54 points on the screen. The laser module projects points with respect to the set points, and a distance error between set points and projected points and angular error are calculated. The distance errors is $1.5 \pm 1.9 \mathrm{~mm}$ (average \pm standard deviation). Maximum error was $7.5 \mathrm{~mm}$. Angular error was smaller than 2 degrees. The laser projection system and its calibration method shows clinically acceptable accuracy and the clinical application is the next step.
\end{abstract}

\section{Introduction}

In many orthopedic surgeries using a C-arm fluoroscopy, surgeons make a surgical plan using fluoroscopic images and have to imagine real positions with respect to positions on them. For instance, surgeon plans osteotomy lines or decides insertion positions of medical screws using fluoroscopic images, and then marks their corresponding position to surgical site. However, it is difficult and experience-depending work to find exact corresponding positions. Doke et al. developed fluoroscopy- 
based laser guidance system, and showed its usefulness from phantom experiments (Doke2015). However, surgeon should insert reference markers to patients and the system required an optical tracking system to track the markers. Though the laser guidance system works accurately, it is expensive and burdensome to use the optical tracking system in an operation room. We have developed a laser projection system, which can project laser on corresponding position to surgical planning drawn at a fluoroscopic image without an optical tracking system.

In this paper, we introduce a spatial calibration method between a laser module and a fluoroscope for the laser projection and evaluate its accuracy with a mimic experimental system.

\section{Materials and Methods}

The experimental system consists of a laser module, a distance measurement unit and a CCD camera as shown in figure 1(a). The laser modules can project arbitrary line on surface by reflecting a point source laser with two galvanometers. A distance measurement unit can measure distance from the laser module to the projection surface, and the camera mimics a fluoroscopy. We have to estimate spatial relation of three coordinates-the laser module, the surface, and the camera- to reflect the arbitrary line drawn on images of the camera to the surface as shown in figure 1(b). The ${ }_{C}^{L} T$ matrix translating the camera coordinate to the laser coordinate is represented in (1), ${ }_{C} T$ is an extrinsic parameter of a camera detecting a chessboard, and ${ }_{W}^{L} T$ is given by putting the chessboard on a special laser pattern from the laser module.

$$
{ }_{C}^{L} T={ }_{W}^{L} T{ }_{C}^{W} T
$$

A calibration phantom for world coordinate is designed by combining a collimator for accurate laser pattern positioning and stainless steel ball arrays for calculation of an extrinsic parameter of a Carm fluoroscopy.

We set a projection plane in 400mm distance from the CCD camera, and set 54 points on the screen. The laser module projects points with respect to the set points, and a distance error between set points and projected points and angular error are calculated.

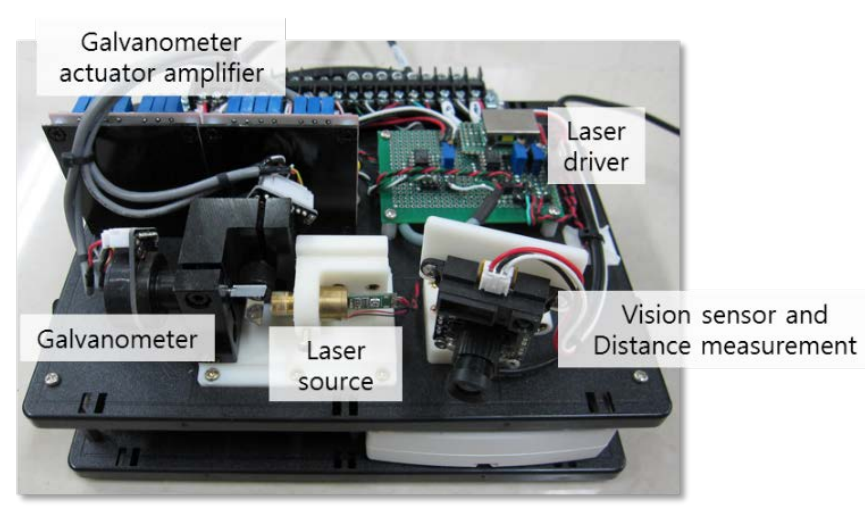

(a)

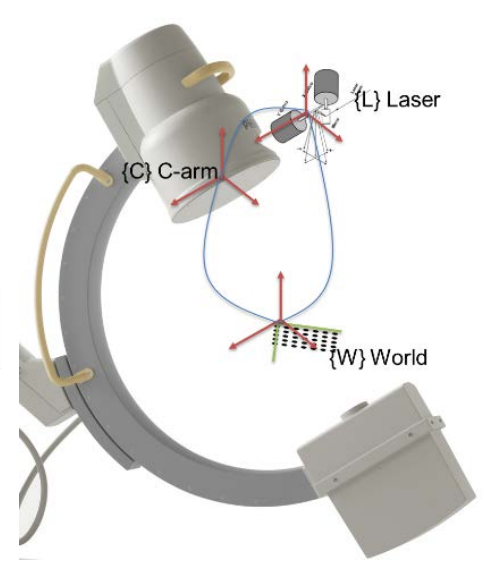

(b)

Figure 1. Laser projection system and its coordinate relation; (a) experimental system configuration, and (b) coordinate relation between a $\mathrm{C}$-arm, a laser module and a world coordinate 


\section{Results}

Figure 2(a) shows the calibration phantom and its fluoroscopic image. Figure 2(b) shows experimental results; red points are the set points and green shows projected laser line. The distance errors is $1.5 \pm 1.9 \mathrm{~mm}$ (average \pm standard deviation). Maximum error was $7.5 \mathrm{~mm}$. It has tendency of increasing error at upper side of the screen. Angular error was smaller than 2 degrees.
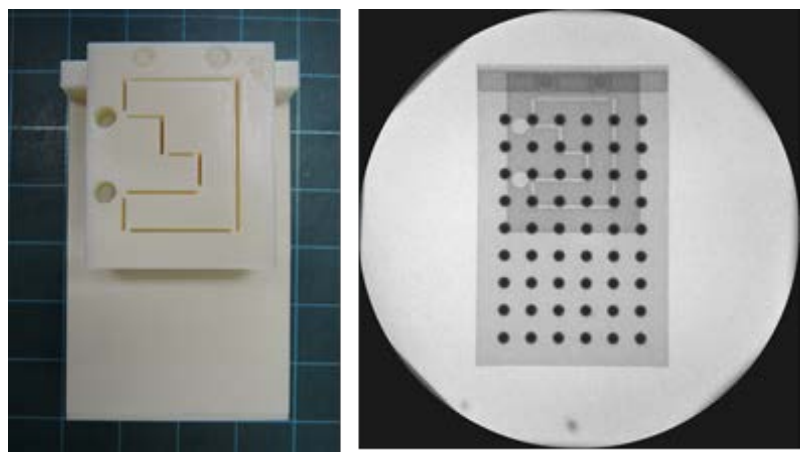

(a)

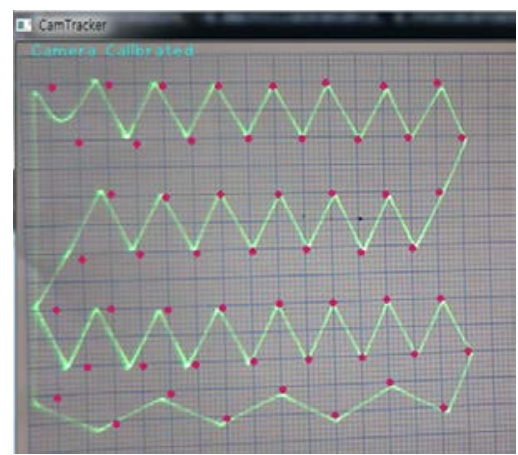

(b)

Figure 2. (a) Calibration phantom and its fluoroscopic image, (b) laser projection results.

\section{Discussion}

We have developed the laser projection system for surgical guidance that shows the arbitrary lines on surgical site corresponding surgical planning drawn at fluoroscopic images. The suggested calibration method shows clinically acceptable accuracy and the clinical application is the next step.

Acknowledgment: This work was supported by the Industrial Strategic Technology Development Program (10063309), funded by the Ministry of Trade, Industry and Energy (MI, Korea).

\section{References}

Takehito Doke and et al., Fluoroscopy-based laser guidance system for linear surgical tool insertion depth control, International Journal of Computer Assisted Radiology and Surgery, vol. 10(3), pp. 275-283, 2015 